\title{
Control Biológico y Respuesta Varietal ante Hongos Patógenos de la Caña de Azucar
}

\author{
Carlos M. Andreu Rodríguez \\ Mabel Pérez Gonzáles
}

\author{
Yolanda Morales Gómez \\ Reinaldo Quiñones Ramos
}

Centro de Investigaciones Agropecuarias (CIAP)

Universidad Central de las Villas, Cuba.

\begin{abstract}
RESUMEN
Los efectos de biocontrol de Trichoderma viride (Ts-III-85) y Gliocladium Virens (Gv02) sobre Fusarium moniliforme (Fm-01) y Thielaviopsis paradoxa (Tp-02) y el comportamiento varietal ante estos hongos patógenos se estudiaron en variedades comerciales de caña de azúcar. Para ello se efectuaron varios ensayos de laboratorio con distintos métodos de tratamiento a las estacas e inoculaciones artificiales de los hongos patógenos. Los resultados mostraron el efecto de biocontrol de los hongos antagonistas sobre los hongos fitopatógenos, los síntomas fueron menores con tratamientos de $T$. viride y $G$. virens en las variedades Jaronú 60/5; Jaronú 64/19 y Cuba 1051/73. La variedad Jaronú 60/5 fue más atacada por $F$. moniliforme y en la Cuba 1051/73 por $T$. paradoxa.
\end{abstract}

Palabras clave: Caña de Azúcar, biocontrol, variedades, Trichoderma, Gliocladium, Fusarium, Thielaviopsis.
The biocontrol effect of Trichoderma viride (Ts-III-85) and Gliocladium virens (Gv-02 upon Fusarium moniliforme (Fm-01) and Thielaviopsis paradoxa ( $\mathrm{Tp}-02)$ were studied in sugarcane varieties as well as the behavior of varieties in the presence of these fungi. In order to obtain the results some laboratory test using different methods of treatment of stakes and by inoculating artificiallt with pathogenic fungi were developed. The results showed the biocontrol effect of the antagonistic fungi upon F. moniliforme and T. paradoxa. For the varieties Jaronú 60/5; Jaronú 64/19 and Cuba 1051/73 treated with T. viride and $G$. virens the symptoms were smaller. The Jaronú $60 / 5$ variety was the one which $F$. moniliforme attacked the most; being Cuba 1051/73 the one mostly attacked by T. paradoxa.

Key words: Sugarcane, biocontrol, varieties, Trichoderma, Gliocladium, Fusarium, Thielaviopsis. 


\section{INTRODUCCION}

Entre los hongos fitopatógenos que originan enfermedades de importancia económica en la caña de azúcar(Saccharum spp) se encuentran Fusarium moniliforme y Thielavipsis paradoxa, los que presentan una gran incidencia sobre variedades comerciales en diferentes países (Hughes et al., 1961, 1964).

Varios investigadores estudiaron los efectos antagónicos in vitro de algunos hongos sobre F. moniliforme y T. paradoxa, los que determinaron la efectividad de cepas para el control biológico de estos hongos (Andreu et al., 1995 a, b; Borras et al., 1995). No obstante, estos efectos sobre las estacas infectadas de variedades comerciales no han sido lo suficientemente abordados con vistas a poder establecer nuevas medidas de lucha fitosanitaria contra estos hongos patógenos.

\section{MATERIALES Y METODOS}

Los estudios se realizaron en el Laboratorio de Fitopatología del Centro de Investigaciones Agropecuarias (CIAP) durante 1994; Se hicieron tres ensayos empleándose estacas sanas de 4 meses y yemas activas de las variedades Jaronú 60/5, Jaronú 64/19 y Cuba 1051/73 de 9 meses de edad.

En el primer ensayo se trataron las estacas por separado con sustratos metabólicos con diluciones de 1:1:00 y 1:1000 y suspensión conidial de T. Viride $\left(4,8 \times 10^{8} \mathrm{esp} / \mathrm{ml}\right)$.

En los ensayos 2 y 3 se usaron tratamientos que consistieron en la inmersión de las estacas de suspensión conidial de T. viride $\left(3 \times 10^{8}\right.$ esp/ml) y G. virens $\left.\left(2 \times 10^{8}\right) \mathrm{esp} / \mathrm{ml}\right)$ de forma independiente y luego se aplicó un disco de PDA de $8 \mathrm{~mm}$ de diámetro con crecimiento de F. moniliforme y en otro caso con T. paradoxa.

Para comprobar la efectividad biológica en los diferentes experimentos se usó un control donde las estacas se sometieron a inoculación artificial con discos micelial en cada estaca y se cubrió con polietileno desinfectado con hipoclorito de sodio al 3\%. Cada tratamiento contó con 10 repeticiones y se incubó a $28{ }^{\circ} \mathrm{C}$. En el primer estudio se evaluó midiendo en cm los síntomas y en los restantes aplicando la siguiente escala:

Grado 0: Sin síntomas en la estaca.

Grado 1: Lesión con menos de $1 \mathrm{~cm}$

Grado2: Lesión de 1-2cm.

Grado 3: Lesión 2-3cm.

Grado 4: Lesión de 3-4

Grado 5: Lesión de 4-5cm.

Grado 6: Lesión de más de $5 \mathrm{~cm}$. 
Se calculó la intensidad del ataque mediante la fórmula de Towsend y Heuberguer (1949) que consistió en:

$$
P=\frac{\text { Suma }(\text { n.v })}{\text { N.K }} 100
$$

Donde:

$\mathrm{P}=$ Intensidad del ataque

$\mathrm{n}=$ número de estacas en cada categoria de ataque

$\mathrm{V}=$ Valor numérico de la escala

$\mathrm{N}=$ número total de estacas evaluadas

$\mathrm{K}$ = máximo valor de la escala

Los resultados obtenidos fueron procesados con análisis de varianza y prueba de MDS.

\section{RESULTADOS Y DISCUSION}

En la Tabla 1 pueden verse los resultados del primer ensayo. Nótese que en las estacas donde no se empleó $T$. viride el largo de la lesión fue significativamente mayor y no se registraron daños cuando se aplicó a las mismas micelio y esporas del hongo antagonista (disco mecelial), lo que impidió la invasión de $F$. moniliforme en los tejidos. A continuación estuvieron los datos de los resultados del empleo de metabolitos y esporas de $T$. viride, los que no se diferenciaron estadísticamente entre sí.

Los resultados del ensayo 2 se exponen en la tabla 2 sección 1, en la que se observa una disminución significativa de los daños por $F$. moniliforme al aplicarse a las estacas de ambas variedades la suspensión conidial de $T$. viride al compararse con los controles. Se denotó además, mayor afectación por el hongo patógeno en la variedad jaronú 60/5, siendo menor en Cuba 1051. Igual resultado ocurrió al analizarse el efecto de biocontrol de T. viride sobre $T$. paradoxa (sección 2). En la variedad Cuba 1051/73 se notó una mayor afectación por el hongo patógeno. Los daños y el afecto de biocontrol en la variedad Cuba 1051/73 fueron iguales desde el punto de vista estadístico, no siendo así al valorar estos resultados en la variedad Jaronú 60/5, la que fue más afectada por F. moniliforme que por T. paradoxa.

\section{Tabla 1.}

Biocontrol de $T$. viride sobre

F. moniliforme en la variedad Jaronú 64/19 en condiciones de laboratorio.

\section{Tratamientos}

Largo de lesión (cm) prom.

\section{Control}

4,17 a

2. Inoculación disco micelial de $T$. viride

3. Aplicación de metabolitos (1:100)

4. Aplicación de metabolitos (1:1000)

$2,57 \mathrm{~b}$ 
Tabla 2 Biocontrol de $T$. viride sobre $F$. moniliforme y

$T$ paradoxa en dos variedades de caña de azúcar (ensayo 2)

\section{Sección 1}

Tratamientos

1. Control $\sin T$. viride

2. Trat. estacas con $T$. viride
Afectación por $F$. moniliforme (\%)

\section{Jaronú 60/5}

6,92 a

$4,89 \mathrm{~b}$

CV: $8,4 \%$

Afectación por T. paradoxa (\%)

\section{Sección 2}

\section{Tratamientos}

1. Control $\sin T$. viride

Jaronú 60/5

2. Trat. Estacas con T. viride $1,70 \mathrm{~b}$

CV: $9,8 \%$
Cuba 1051/73

4,48 a

$2,47 \mathrm{~b}$

CV: $7,1 \%$
En todos los casos medias con letras desiguales difieren Significativamente al nivel P 0,01

En la tabla 3 se expone los resultados del ensayo 3. Obsérvese el efecto de biocontrol ejercido por $G$. virens sobre $F$. moniliforme (secc.1) y T. paradoxa ( $\operatorname{secc} .2$ ), donde los síntomas en las variedades fueron significativamente menores usadas como material de siembra. El mayor porcentaje de afectación se registró en cada control.

Tabla 3 Biocontrol de $G$. virens sobre $F$. moniliforme y T.paradoxa en variedades de caña de azúcar (ensayo 3 ).

\section{Sección 1}

1. Control $\sin G$. virens

2.Trat. estacas con $G$. virens

6,87 a

$4,22 \mathrm{~b}$

CV: $2,6 \%$

Afectación por T. paradoxa $(\%)$

\section{Sección 2}

\section{Tratamientos}

1. Control $\sin G$. virens

2. Trat estacas con $G$. virens 1,51 b

\section{Jaronú 60/5}

\section{Tratamientos}

\section{Jaronú 60/5}

$$
\text { CV: } 6,1 \%
$$

2,79 a Cuba 1051/73

3,94 a

$1,72 \mathrm{~b}$

CV: $1,5 \%$
En todos los casos medias con letras desiguales difieren significativamente $\mathrm{P} 0,01$
Al valorar el comportamiento

de las variedades ante $F$. moniliforme y $T$. paradoxa pudo verse como tendencia, que la variedad Jaronú $60 / 5$ fue más afectada por $F$. moniliforme que Cuba 1051/73, y esta variedad a su vez, fue más susceptible ante T. paradoxa.

Los resultados en estos ensayos evidenciaron la efectividad biológica de $T$. viride $\mathrm{y}$ $G$. virens sobre estos hongos fitopatógenos a los que se les disminuyó la capacidad de infección en los tejidos parenquimatosos y vasculares de las estacas.

Pudo observarse además que $T$. viride y $G$. virens fueron capaces de crecer y esporular en zonas de los tejidos al cabo de los 9 días de realizar los tratamientos con estos microorganismos.

Al respecto Andreu et al., (1995 a, b) determinaron el nivel competitivo y de hiperparasitismo in vitro de estas cepas de hongos antagonistas sobre $F$. moniliforme (Fm 01) y T. paradoxa (Tp-02). Otros investigadores aseveraron que estos hongos antagonistas 
emitieron metabolitos y enzimas que afectaron el crecimiento y esporulación de varias especies de hongos fitopatogenos, lo que permitió valorarlos útiles como controles biológicos (Backman y Rodríguez-Kabana, 1975; Cherif y Benhamou, 1990; Lumsden et al., 1992).

\section{CONCLUSIONES}

1.- El empleo de diferentes métodos de tratamientos a las estacas de variedades de caña de azúcar con Trichoderma viride (Ts-III-85) implicó la reducción de los síntomas originados por Fusarium moniliforme y Thielaviopsis paradoxa.

2.- La aplicación de la suspensión conidial de Gliocladium virens a las estacas de caña de azúcar disminuyó significativamente las afectaciones a causa de $F$. moniliforme y T. paradoxa.

3.- Se determinó como tendencia, que la verdad Jaronú $60 / 5$ fue más afectada por Fusarium moniliforme y la variedad Cuba 1051/73 presentó mayores síntomas por T. paradoxa.

\section{BIBLIOGRAFIA}

ANDREU, C.M. et al. Efecto antagónico in vitro de Trichoderma spp y Gliocladium virens sobre Fusarium moniliforme. En : Revista Centro Azúcar, Cuba (en prensa), (1995 a).

Actividad in vitro de Trichoderma viride y Gliocladium virens sobre Thielaviopsis paradoxa. Revista Centro Azúcar, Cuba (en prensa), (1995 b).

BACKMAN, P. A. and RODRIGUEZ-KABANA. A system for the growth and delivery of biological control agents to soil.

En : Phytopathology 65 (1971) p. 819-821.

BORRAS, O. et al., Utilización de especies de Trichoderma en el control biológico de Fusarium moniliforme, Chalara paradoxa y Phytopathora nocotianae var parasitica. Res. Seminario científico ISCAH, habana, 1995.

CHERIF, M. and BENHAMOU, N. Cytochemical aspects of chitin breakdown during the parasitic action of Trichoderma spp on Fusarium oxysporum. Phytopathology 80 (1990) p. 1406.

HUGHES, C.B.; ABBOTT, E.V. and WISMER, C.A. Sugar cane diseases of the world. Habana, DE Pueblo y Educ. 1961, Vol Y p. 99-101.

p. $188-205$. , Habana : Pueblo y Educ., 1964. Vol II

LUMSDEN, R.D. et al. Rapid quanification of metabolites from Gliocladium by video imaging. En : Phytopathology. 82 (1992) p. 1127.

TOWSEND, D. and HEUBERGUER, J. Method for estimating losses caused by diseases with fungicides experiments. En : Plant Disease Reporter 27 (1949) p.340-343. 